\title{
Weighted Sharing and Uniqueness of Entire Functions Concerning Differential Polynomials
}

\author{
Nintu Mandal ${ }^{1}$ \\ ${ }^{I}$ (Department of Mathematics, Chandernagore College, Chandernagore, Hooghly, West Bengal, India)
}

\begin{abstract}
Using Nevanlinna value distribution theory, we study weighted sharing and uniqueness of entire functions concerning differential polynomials and obtain some results which improve the recent results due to Waghamore and Anand[1].
\end{abstract}

Keywords: Differential Polynomials, Entire Functions, Uniqueness, Value Distribution Theory, Weighted Sharing.

\section{Introduction and Definitions}

In this paper we use the standard definitions and notations of the value distribution theory [2]. Let $f$ and $g$ be two nonconstant meromorphic functions and $a \in \mathbb{C} \cup\{\infty\}$. We say that $f$ and $g$ share the value $a \mathrm{CM}$ (counting multiplicities) if the zeros of $f-a$ and $g-a$ coincide in locations and multiplicities. If we do not consider the multiplicities, we say that $f$ and $g$ share the value $a \mathrm{IM}$ (ignoring multiplicities). We say that $f$ and $g$ share a function $h \mathrm{CM}$ or IM if and only if $f-h$ and $g-h$ share $0 \mathrm{CM}$ or IM. For $a \in \mathbb{C} \cup\{\infty\}$, we denote by $N(r, a ; f)$ the counting function of all the $a$-points of $f$ and by $\bar{N}(r, a ; f)$ the corresponding one for which the multiplicity is not counted. For a positive integer $k$, we denote by $N_{k}(r, a ; f)$ the counting function of $a$-points of $f$, where an $a$-point of multiplicity $m$ is counted $m$ times if and only if $m \leq k$ and $k$ times if and only if $m>k$.We denote by $S(r, f)$ any quantity satisfying $S(r, f)=o\{T(r, f)\}$ as $r \rightarrow \infty$ possibly outside a set of finite linear measure. If $T(r, \alpha)=S(r, f)$ for a meromorphic function $\alpha=\alpha(z)$, then $\alpha$ is called small function of $f$.

Definition 1.1. [3]Let $k$ be a nonnegative integer or infinity. For $a \in \mathbb{C} \cup\{\infty\}$, we denote by $E_{k}(a ; f)$ the set of all $a$-points of $f$ where an $a$-point of multiplicity $m$ is counted $m$ times if $m \leq k$ and $k+1$ times if $m>k$. If $E_{k}(a ; f)=E_{k}(a ; g)$, we say that $f$ and $g$ share the value $a$ with weight $k$.

The definition implies that if $f$ and $g$ share a value $a$ with weight $k$ then $z_{0}$ is a zero of $f-a$ with multiplicity $m(\leq k)$ if and only if it is a zero of $g-a$ with multiplicitym $(\leq k)$ and $z_{0}$ is a zero of $f-a$ with multiplicity $\mathrm{m}(>\mathrm{k})$ if and only if it is a zero of $g-a$ with multiplicity $n(>k)$, where $m$ is not necessarily equal to $\mathrm{n}$.

We write $f$ and $g$ share $(a, k)$ to mean that $f$ and $g$ share the value $a$ with weight $k$. Clearly if $f$ and $g$ share $(a, k)$ then $f$ and $g$ share $(a, p)$ for all integers $p, 0 \leq p<k$. Also we note that $f$ and $g$ share a value $a$ IM or $\mathrm{CM}$ if and only if $f$ and $g$ share $(a, 0)$ or $(a, \infty)$ respectively.

If $\alpha$ is a small function of $f$ and $g$, then $f$ and $g$ share $\alpha$ with weight $k$ means that $f-\alpha$ and $g-\alpha$ share the value 0 with weight $k$.

In 1996 Fang and Hua [4] proved the following theorem:

Theorem A. [4]Let $f$ and $g$ be two nonconstant entire functions. Also let $n \geq 6$ be a positive integer. If $f^{n} f^{\prime}$ and $g^{n} g^{\prime}$ share the value $1 \mathrm{CM}$, then one of the following holds

(i) $f(z)=c_{1} e^{c z} ; g(z)=c_{2} e^{-c z}$; where $c_{1}, c_{2}$ and $c$ are three constants satisfying $\left(c_{1} c_{2}\right)^{n+1} c^{2}=-1$.

(ii) $f=k g$ for a constant $k$ such that $k^{n+1}=1$.

In 2001, M. L. Fang and W. Hong [5] obtained the following result.

Theorem B. [5]Let $f$ and $g$ be two transcendental entire functions, $n \geq 11$ an integer. If $f^{n}(f-1) f^{\prime}$ and $g^{n}(g-1) g^{\prime}$ share the value $1 \mathrm{CM}$, then $f \equiv g$.

In 2013, S. S. Bhoosnurmath and V. L. Pujari [6] obtained the following result.

Theorem C. [6]Let $f$ and $g$ be two nonconstant entire functions, $n \geq 7$ an integer. If $f^{n}(f-1) f^{\prime}$ and $g^{n}(g-$ 1) $g^{\prime}$ share $z$ CM, then $f \equiv g$.

Recently H. P. Waghamore and S. Anand [1] proved the following theorem:

Theorem D. [1]Let $f$ and $g$ be two nonconstant entire functions and $n, m$ be positive integers such that $n \geq m+6$. If $f^{n}(f-1)^{m} f^{\prime}$ and $g^{n}(g-1)^{m} g^{\prime}$ share $z \mathrm{CM}$, then $f \equiv g$.

Since

$$
\begin{aligned}
f^{n}(f-1)^{m} f^{\prime}= & \frac{1}{n+1}\left(f^{n+1}\right)^{\prime}(f-1)^{m} \\
& =\left[f^{n+1}\left(\frac{{ }^{n} C_{m}}{n+m+1} f^{m}-\frac{{ }^{n} C_{m-1}}{n+m} f^{m-1}+\cdots+\frac{{ }^{n} C_{0}}{n+1}(-1)^{m}\right)\right] .
\end{aligned}
$$


Therefore it is natural to consider the uniqueness of meromorphic functions concerning more general kind differential polynomial, such as $\left[f^{n} L(f)\right]^{(k)}$, where

$$
L(z)=\alpha_{m} z^{m}+\alpha_{m-1} z^{m-1}+\cdots+\alpha_{0}
$$

and $\alpha_{m} \neq 0, \alpha_{m-1}, \alpha_{m-2}, \ldots, \alpha_{0} \neq 0$ are complex constants.

In this paper we prove the following result

Theorem 1.1.Let $f$ and $g$ be two transcendental entire functions, $n \geq 1, m \geq 1, \mathrm{k} \geq 0$, be three integers such that $n>2 k+m+4$. If $\left[f^{n} L(f)\right]^{(k)}$ and $\left[g^{n} L(g)\right]^{(k)}$ share $(z, 2)$, where $L(z)$ is defined as in $(1.1)$, then one of the following cases holds:

(i) $f=e^{\beta_{1}}$ and $g=e^{\beta_{2}}$; where $\beta_{1}$ and $\beta_{2}$ are nonconstant entire functions.

(ii) $f=\operatorname{tg}$ for a constant $t$ such that $t^{p}=1$, where $p=n+m-i, \alpha_{m-i} \neq 0$ for some $i=0,1, \ldots$, $m$.

(iii) $f$ and $g$ satisfy algebraic euation $Q\left(x_{1}, x_{2}\right)=0$, where

$$
Q\left(x_{1}, x_{2}\right)=x_{1}^{n}\left(\alpha_{m} x_{1}^{m}+\alpha_{m-1} x_{1}^{m-1}+\cdots+\alpha_{0}\right)-x_{2}^{n}\left(\alpha_{m} x_{2}^{m}+\alpha_{m-1} x_{2}^{m-1}+\cdots+\alpha_{0}\right)
$$

\section{Lemmas}

In this section we present some lemmas which are required in the sequel.

Lemma 2.1. [7] Let $f$ be a nonconstant meromorphic function and let $\alpha_{l} \neq 0, \alpha_{l-1}, \alpha_{l-2}, \ldots, \alpha_{0}$ be small functions with respect to $f$. Then

$$
T\left(r, \alpha_{l} f^{l}+\alpha_{l-1} f^{l-1}+\cdots+\alpha_{0}\right)=l T(r, f)+S(r ; f) \text {. }
$$

Lemma 2.2. [3] Let $f$ and $g$ be two nonconstant meromorphic functions sharing $(1,2)$. Then one of the following cases holds:

(i) $f \equiv g$.

(ii) $T(r) \leq N_{2}(r, 0 ; f)+N_{2}(r, 0 ; g)+N_{2}(r, \infty ; f)+N_{2}(r, \infty ; g)+S(r)$

(iii) $f g \equiv 1$,

where $T(r)=\max \{T(r, f), T(r, g)\}$ and $S(r)=o\{T(r)\}$.

Lemma 2.3. [8] Let $f$ be a nonconstant meromorphic function and $k$ be a positive integer. Also let $c$ be a nonzero finite complex number. Then

$$
T(r, f) \leq N_{k+1}(r, 0 ; f)+\bar{N}\left(r, 0 ; f^{(k)}-c\right)+\bar{N}(r, \infty ; f)-N_{0}\left(r, 0 ; f^{k+1}\right)+S(r, f),
$$

where $N_{0}\left(r, 0 ; f^{k+1}\right)$ denotes the counting function of the zeros of $f^{(k+1)}$ which are not zeros of $f\left(f^{(k)}-c\right)$.

Lemma 2.4. [9] Let $f$ be a nonconstant meromorphic function and $p, k$ be two positive integers. Then

$$
N_{p}\left(r, 0 ; f^{(k)}\right) \leq T\left(r, f^{(k)}\right)-T(r, f)+N_{p+k}(r, 0 ; f)+S(r ; f),
$$

and

$$
N_{p}\left(r, 0 ; f^{(k)}\right) \leq N_{p+k}(r, 0 ; f)+k \bar{N}(r, \infty ; f)+S(r ; f) .
$$

Lemma 2.5. Let $f$ and $g$ be two nonconstant entire functions. Also let $F=\left[f^{n} L(f)\right]^{(k)}$ and $G=\left[g^{n} L(g)\right]^{(k)}$, where $L(z)$ is defined as in (1.1). If there exists three nonzero constants $\lambda_{1}, \lambda_{2}, \lambda_{3}$, such that $\lambda_{1} F+\lambda_{2} G=\lambda_{3}$ then $n \leq 2 k+m+2$.

Proof. Since $f$ and $g$ are entire functions therefore by Lemma 2.1, Lemma 2.3 and Lemma 2.4 we have

$(n+m) T(r, f) \leq N_{k+1}\left(r, 0 ; f^{n} L(f)\right)+\bar{N}\left(r, 0 ; F-\frac{\lambda_{3}}{\lambda_{1}}\right)+S(r, f)$

$\leq N_{k+1}\left(r, 0 ; f^{n} L(f)\right)+\bar{N}(r, 0 ; G)+S(r, f)$ $\leq N_{k+1}\left(r, 0 ; f^{n} L(f)\right)+N_{k+1}\left(r, 0 ; g^{n} L(g)\right)+k \bar{N}\left(r, 0 ; g^{n} L(g)\right)+S(r, f)+S(r, g)$

$\leq N_{k+1}\left(r, 0 ; f^{n} L(f)\right)+N_{k+1}\left(r, 0 ; g^{n} L(g)\right)+S(r, f)+S(r, g)$

$$
\leq(k+m+1) T(r, f)+(k+m+1) T(r, g)+S(r, f)+S(r, g) \ldots \ldots \ldots
$$

Similarly we have

$$
(n+m) T(r, f) \leq(k+m+1) T(r, g)+(k+m+1) T(r, f)+S(r, f)+S(r, g) \ldots \ldots \ldots
$$

From (2.1) and (2.2) we have

$$
(n-2 k-2-m)\{T(r, f)+T(r, g)\} \leq S(r, f)+S(r, g) \ldots \ldots \ldots
$$

From (2.3) we get $n \leq 2 k+m+2$.

\section{Proof of the Main Result}

Proof of Theorem 1.1:

Let $F=f^{n} L(f), G=g^{n} L(g), F_{1}=\left[f^{n} L(f)\right]^{(k)}, G_{1}=\left[g^{n} L(g)\right]^{(k)}, F^{*}=\frac{F}{z}$ and $G^{*}=\frac{G}{z}$. Clearly $F^{*}$ and $G^{*}$ share $(1,2)$ and $\infty$ IM. Hence by Lemma 2.2one of the following holds:

(i) $F^{*} \equiv G^{*}$.

(ii) $T(r) \leq N_{2}\left(r, 0 ; F^{*}\right)+N_{2}\left(r, 0 ; G^{*}\right)+N_{2}\left(r, \infty ; F^{*}\right)+N_{2}\left(r, \infty ; G^{*}\right)+S(r)$

(iii) $F^{*} G^{*} \equiv 1$,

where $T(r)=\max \left\{T\left(r, F^{*}\right), T\left(r, G^{*}\right)\right\}$ and $S(r)=o\{T(r)\}$. 
So we have to consider the following cases.

Case I: $F^{*} \equiv G^{*}$.

Integrating we have

$$
\left[f^{n} L(f)\right]^{(k-1)} \equiv\left[g^{n} L(g)\right]^{(k-1)}+c_{k-1},
$$

where $c_{k-1}$ is a constant. If $c_{k-1} \neq 0$, using Lemma 2.5 it follows that $n \leq 2 k+m$, a contradiction. Hence $c_{k-1}=0$. Repeating the same process for $k-1$ times, we get

$$
f^{n} L(f) \equiv g^{n} L(g) \ldots \ldots \ldots
$$

From (3.1) we have

Let $t=\frac{f}{g}$.

If $t$ is a constant then substituting $f=\operatorname{tg}$ in (3.2) we get

$$
\alpha_{m} g^{n+m}\left(t^{n+m}-1\right)+\alpha_{m-1} g^{n+m-1}\left(t^{n+m-1}-1\right)+\cdots+\alpha_{0} g^{n}\left(t^{n}-1\right)=0,
$$

which implies that $t^{p}=1$, where $p=n+\mathrm{m}-\mathrm{i}, \alpha_{m-i} \neq 0$ for some $i=0,1,2, \ldots, m$.

Hence $f \equiv t g$, for a constant $t$, such that $t^{p}=1$, where $p=n+\mathrm{m}-\mathrm{i}, \alpha_{m-i} \neq 0$ for some $i=0,1,2, \ldots, m$.

If $t$ is not a constant, then by (3.3) $f$ and $g$ satisfy the algebraic equation $Q\left(x_{1}, x_{2}\right)=0$, where

$$
Q\left(x_{1}, x_{2}\right)=x_{1}^{n}\left(\alpha_{m} x_{1}^{m}+\alpha_{m-1} x_{1}^{m-1}+\cdots+\alpha_{0}\right)-x_{2}^{n}\left(\alpha_{m} x_{2}^{m}+\alpha_{m-1} x_{2}^{m-1}+\cdots+\alpha_{0}\right)
$$

Case II: In this case we have

$$
T(r) \leq N_{2}\left(r, 0 ; F^{*}\right)+N_{2}\left(r, 0 ; G^{*}\right)+N_{2}\left(r, \infty ; F^{*}\right)+N_{2}\left(r, \infty ; G^{*}\right)+S(r),
$$

where $T(r)=\max \left(T T\left(r, F^{*}\right), T\left(r, G^{*}\right)\right\}$ and $S(r)=o\{T(r)\}$. Without loss of generality, we suppose that $T(r, f) \leq T(r, g), r \in I$, where $I$ is a set of finite measure. By Lemma 2.1 and Lemma 2.4 we get

That is

$$
N_{2}\left(r, 0 ; F_{1}\right) \leq T\left(r, F_{1}\right)-(n+m) T(r, f)+N_{2+k}(r, 0 ; F)+S(r, F)
$$

$$
N_{2}\left(r, 0 ; F_{1}\right) \leq T\left(r, F_{1}\right)-T(r, F)+N_{2+k}\left(r, 0 ; f^{n} L(f)\right)+S(r, f) \ldots \ldots \ldots
$$

Since $f$ and $g$ are transcendental using Lemma 2.1 we have from (3.4)

$T\left(r, F_{1}\right) \leq N_{2}\left(r, 0 ; F_{1}\right)+N_{2}\left(r, 0 ; G_{1}\right)+N_{2}\left(r, \infty ; F_{1}\right)+N_{2}\left(r, 0 ; G_{1}\right)+S\left(r, F_{1}\right)+S\left(r, G_{1}\right)$

$\leq N_{2}\left(r, 0 ; F_{1}\right)+N_{2}\left(r, 0 ; G_{1}\right)+S(r, f) \ldots \ldots \ldots$

Using Lemma 2.4 from (3.5) and (3.6) we have

$$
\begin{aligned}
(n+m) T(r, f) \leq N_{2}(r, 0 ; & \left.G_{1}\right)+N_{2+k}\left(r, 0 ; f^{n} L(f)\right)+S(r, f) \\
& \leq N_{2+k}\left(r, 0 ; g^{n} L(g)\right)+N_{2+k}\left(r, 0 ; f^{n} L(f)\right)+S(r, f) \\
& \leq(2 m+2 k+4) T(r, f)+S(r, f),
\end{aligned}
$$

which contradicts with $n>m+2 k+4$.

Case III: $F^{*} G^{*} \equiv 1$. Thatis $\left[f^{n} L(f)\right]^{(k)}\left[g^{n} L(g)\right]^{(k)} \equiv z^{2}$. Suppose, if possible, that $z_{0}$ is a zero of $f$ of order $p$, then $z_{0}$ must be a zero of $\left[f^{n} L(f)\right]^{(k)}$ of order $n p-k$. Since $n>k+2$ therefore $z_{0}$ must be a zero of $z^{2}$ with the order at least 3. This is impossible. Therefore $f$ has no zero. Hence $f=e^{\beta_{1}}$, where $\beta_{1}$ is a nonconstant entire function. Similarly we can prove that $g=e^{\beta_{2}}$, where $\beta_{2}$ is a nonconstant entire function.

This proves the theorem.

\section{Acknowledgements}

The Authorwould like to thank the UGC (ERO), India for financial support vide UGC MRP No.PSW- 105/1415 (ERO) dated $26^{\text {th }}$ March, 2015.

\section{References}

[1] H. P. Waghamore and S. Anand, Generalization of Uniqueness of Meromorphic Functions Sharing Fixed Point, Appl. Math., 2016, 7, pp. 939-952

[2] W.K.Hayman,Meromorphic Functions, The Clarendon Press, Oxford (1964).

[3] I. Lahiri, Weighted value sharing and uniqueness of meromorphic functions, Complex Var. Theory Appl., 2001, 46, pp.241-253.

[4] M. L. Fang and X. H. Hua, Entire functions that share one value, J. Nanjing Univ. Math. Biquarterly, 1996, 13, pp. 44-48.

[5] M.L. Fang and W. Hong, A unicity theorem for entire functions concerning differential polynomials, Indian J. Pure Appl. Math., 2001, 32, pp.1343-1348.

[6] S.S. Bhoosnurmath and V. L. Pujari, Uniqueness of Meromorphic Functions Sharing Fixed Point, Int. J. Analysis, 2013, Article ID: $538027,12 p$

[7] H. X. Yi and C. C. Yang,Uniqueness theory of meromorphic functions, Science Press, Beijing, 1995.

[8] L. Yang,Value distribution theory, Berlin Spring-Verlag, 1993.

[9] W. C. Lin and H. X. Yi, Uniqueness theorems for meromorphic functions, Indian. J. Pure Appl. Math., 2004, 35, pp.121-132. 